УДК $004.942+76$

\author{
Ю. Є. Боярінова ${ }^{1,2}$, Я. О. Каміновський ${ }^{1}$ \\ ${ }^{1}$ Інститут проблем реєстрації інформації НАН України \\ вул. М. Шпака, 2, 03113 Київ, Україна \\ ${ }^{2}$ Національний технічний університет України \\ «Київський політехнічний інститут імені Ігоря Сікорського» \\ Проспект Перемоги, 37, 03113 Київ, Україна
}

\title{
Особливості побудови представлень експоненціальних функцій у гіперкомплексних числових системах високих вимірностей засобами пакету гіперкомплексних обчислень
}

Розглянуто структуру алгоритму побудови представлення експоненціальної функиії у гіперкомплексних числових системах (ГЧС) високої вимірності методом асоиійованої системи лінійних диференщіальних рівнянь. Наведено необхідні короткі відомості про програмний комплекс гіперкомплексних обчислень (ПКГО), за допомогою якого проведено необхідні громіздкі операчії над символьними виразами при побудові представлення експоненти в ГЧС п'ятої вимірності. Робота супроводжується фрагментами програм у середовищі ПКГО і результатами символьних обчислень.

Ключові слова: гіперкомплексна числова система, представлення функцій, експонента, характеристичне число, системи комп 'ютерної алгебри, алгебраӥчна операчія, таблиия Келі.

Вступ

Один із творців гіперкомплексних чисел В.Р. Гамільтон першим запропонував конструктивне визначення нелінійних трансцендентних функцій від гіперкомплексних змінних. У роботі [1] він пропонує визначити експоненціальну функцію від кватерніонної змінної $q$ як суму степеневого ряду:

$$
\operatorname{Exp}(q)=\sum_{s=0}^{\infty} \frac{q^{s}}{s !} .
$$

3 часом цей підхід був узагальнений на інші трансцендентні функції гіперкомплексної змінної: тригонометричні синус і косинус, гіперболічні синус і косинус та інші, і зараз є загальноприйнятим [2]. Після Гамільтона в багатьох роботах про-

(C) Ю. Є. Боярінова, Я. О. Каліновський 
ведено дослідження з побудови зображення експоненціальної функції від кватерніона. Для цього використовуються різні методи, які базуються на властивостях кватерніонів $[2,3]$. Також побудовано зображення інших трансцендентних функцій кватерніона: логарифмічної функції, тригонометричних синуса і косинуса [3].

Ці функції знайшли важливе використання не тільки в наукових застосуваннях: фізиці, механіці, а і в технічних: орієнтації твердого тіла в просторі, гіроскопії, робототехніці та ін. Задача побудови зображень нелінійних функцій від гіперкомплексного змінного зводиться до їхнього визначення з точки зору структури обчислень над гіперкомплексним аргументом і зображення їх у вигляді гіперкомплексної функції, яка має вигляд

$$
F(X)=\sum_{i=1}^{n} f_{i}\left(x_{1}, \ldots, x_{n}\right) e_{i},
$$

де $X=\sum_{i=1}^{n} x_{i} e_{i}, e_{i}-$ базисні елементи гіперкомплексної числової системи $\Gamma$, а $f_{i}\left(x_{1}, \ldots, x_{n}\right), i=1, \ldots, n$ - дійсні функції. Знання законів виконання алгебраїчних операцій у гіперкомплексних числових системах (ГЧС) дозволяє при визначенні лінійних або нелінійних функцій побудувати їхнє зображення у вигляді гіперкомплексної функції (2). Побудова зображення трансцендентних функцій від гіперкомплексного аргументу зводиться до зображення рядів типу (1) у вигляді гіперкомплексних функцій (2). У деяких простих випадках це можна зробити безпосередньо. Але в загальному випадку потребує розробки спеціальних методів [4-8]. Метою роботи є вирішення деяких алгоритмічних проблем, які виникають при моделюванні представлень експоненціальних функцій у ГЧС високих вимірностей

\section{Побудова преАставлення експоненти віА гіперкомплексної змінної за допомогою асоційованої системи диференціальних рівнянь}

Побудувати представлення експоненти від гіперкомплексної змінної безпосередньо по (1) у вигляді (2) дуже важко. Особливі труднощі виникають при побудові експонент в неканонічних ГЧС і ГЧС великих вимірностей. Авторами був розроблений узагальнений метод побудови [4-8], який можна застосувати в принципі для будь-якої скінченновимірної ГЧС. Він полягає в наступному.

Позначимо вектор-стовпці, складені з компонентів гіперкомплексних змінних, великими латинськими літерами з рискою, наприклад:

$$
\bar{X}=\left(x_{1}, \ldots, x_{n}\right)^{T} .
$$

Представлення експоненти в системі $\Gamma(e, n)$ від змінної $M \in \Gamma(e, n)$, яке будемо позначати $\operatorname{Exp}(M), \epsilon$ часткове рішення гіперкомплексного лінійного диференціального рівняння

$$
\dot{X}=M X
$$

за початкової умови

$$
\operatorname{Exp}(0)=\varepsilon,
$$


де $\varepsilon$ - одиничний елемент системи.

Для побудови рішення гіперкомплексного лінійного диференціального рівняння (4) його необхідно представити у векторно-матричній формі. При цьому

$$
\dot{\bar{X}}=\left(\dot{x}_{1}, \ldots, \dot{x}_{n}\right)^{T}
$$

а вектор-стовпець $\overline{M X}$, отриманий з гіперкомплексного числа $M X$, можна представити у вигляді матричного добутку деякої матриці $\Psi$ розміром $n \times n$, елементи якої $\epsilon$ лінійні комбінації компонентів гіперкомплексного числа $M$, на вектор-стовпець $\bar{X}$ :

$$
\overline{M X}=\Psi \bar{X} .
$$

Тоді гіперкомплексне рівняння (4) перетвориться в систему з $n$ лінійних диференціальних рівнянь першого порядку від дійсних змінних, яка називається асоційованою (з даною ГЧС) системою лінійних диференціальних рівнянь:

$$
\dot{\bar{X}}=\Psi \bar{X} .
$$

Далі, як при звичній процедурі розв'язування лінійних диференціальних рівнянь необхідно знайти характеристичні числа $\lambda_{1}, \ldots, \lambda_{n}$ матриці $\Psi$, тобто вирішити характеристичне рівняння

$$
\Psi-\lambda E=0 .
$$

Таким чином, характеристичні числа $\lambda_{1}, \ldots, \lambda_{n}$ будуть залежати від гіперкомплексного $M$ числа.

Загальні рішення системи (8) $\bar{X}\left(t, C_{1}, \ldots, C_{n}, \bar{M}\right)$, залежать від $n$ довільних констант і компонентів гіперкомплексного числа $M$. Значення довільних постійних встановлюються за допомогою початкової умови (5). Компоненти вектор-стовпця $\bar{X}$ рішення і будуть компонентами експоненти від гіперкомплексного числа $M$ :

$$
\operatorname{Exp}(M)=\sum_{i=1}^{n} \bar{x}_{i} e_{i}
$$

Метод побудови представлень експоненти від гіперкомплексної змінної за допомогою асоційованої системи лінійних диференціальних рівнянь досить легко формалізується для побудови алгоритмів і програм в системах символьних обчислень. У цьому процесі основною перешкодою є громіздкість гіперкомплексних виразів у символьній формі, особливо при великих вимірностях застосовуваних ГЧС. Для уникнення цих труднощів потрібна розробка та застосування спеціальних засобів проведення обчислень з гіперкомплексними виразами.

\section{Основні відомості про пакет гіперкомплексних обчислень}

Майже всі системи комп'ютерної алгебри (СКА) мають засоби оперування 3 комплексними числами в символьному або чисельному виглядах. Деякі СКА, а саме: Matlab, Mathcad, Mathematica, Maple, MuPAD, S-PLUS та ін. [9, 10], мають 
засоби оперування з обмеженим набором гіперкомплексних числових систем, такими як кватерніони, октоніони, кліфордові алгебри та деякі інші. Крім того, перелік операцій у цих СКА невеликий. Така ситуація потребує для вирішення конкретних задач з використанням гіперкомплексних числових систем створення спеціального алгоритмічно-програмного забезпечення. Тому протягом останніх років у відділі Спеціальних засобів моделювання ІПРІ НАН України проводяться роботи зі створення алгоритмічно-програмних засобів моделювання задач з використанням гіперкомплексних числових систем. Ці засоби являють собою пакет програм і процедур, створених на основі СКА Maple. Найбільш повний опис цього пакету представлено в роботі [11].

Так як система комп'ютерної алгебри Марle дозволяє створювати спеціалізовані пакети різних обчислювальних процедур, то створений програмний комплекс гіперкомплексних обчислень (надалі - ПКГО) являє собою пакет, що має свій ідентифікатор. ПКГО можна викликати, приєднувати до програми та транспортувати на інші комп'ютери. 3 процедур ПКГО можна формувати програми обчислень, використовуючи засоби алгоритмічної мови Maple.

ПКГО може бути інстальований на будь-якому комп'ютері з операційною системою Windows і системою комп'ютерних обчислень Maple не нижче 5-ї версії. ПКГО відкритий для поповнення новими процедурами та редагування існуючих процедур будь-яким користувачем, що володіє Maple.

Багато уваги при розробці ПКГО було приділено способам і структурам представлення даних. Як було зазначено вище, ПКГО призначений для оперування 3 даними в гіперкомплексному вигляді. Як відомо, загальний вигляд гіперкомплексного числа такий:

$$
A=a_{1} e_{1}+a_{2} e_{2}+\cdots+a_{n} e_{n},
$$

де $n$ - вимірність ГЧС; $a_{i}$ - алгебраїчні вирази; $e_{i}$ - елементи базису ГЧС («уявні одиниці»).

Таку форму гіперкомплексного числа будемо називати натуральною. Як показує досвід, оперувати з гіперкомплексними числами в натуральній формі досить незручно. Це пов'язано з тим, що різні операції виконуються з коефіцієнтами при базисних елементах, які потрібно виділяти й ідентифікувати. Розглянемо для прикладу таку просту операцію як додавання гіперкомплексних чисел. Нехай поряд 3 (11) є число $B$ такого ж вигляду. Якщо в рамках Maple використовувати операцію додавання, то вийде наступне:

$$
C=A+B=a_{1} e_{1}+a_{2} e_{2}+\cdots+a_{n} e_{n}+b e_{1}+b_{2} e_{2}+\cdots+b_{n} e_{n} .
$$

Вираз у правій частині (12) не є натуральною формою гіперкомплексного числа. У ньому потрібно зробити приведення подібних доданків. У системі Maple $\epsilon$ команда приведення подібних доданків - collect, але при ії застосуванні необхідно вказувати змінну, за якою проводиться приведення подібних. Тому в даному випадку доведеться $n$ раз застосувати команду collect, вказуючи кожен раз, з якої змінної проводиться приведення подібних:

$$
\left.C=\operatorname{collect}\left(\left(\text { collect } \ldots(A+B), e_{1}\right), \ldots\right), e_{n}\right)=\left(a_{1}+b_{1}\right) e_{1}+\cdots+\left(a_{n}+b_{n}\right) e_{n} .
$$


Як видно, (13) - складна конструкція, особливо при великих вимірностях ГЧС. Таких незручностей, що пов'язані з використанням натуральної форми подання гіперкомплексних чисел, дуже багато.

У системі Maple є засоби, що дозволяють позбутися цих і багатьох інших незручностей, пов'язаних з використанням натуральної форми подання гіперкомплексних чисел. Справа в тому, що в натуральній формі представлення гіперкомплексних чисел важливі тільки коефіцієнти при елементах базису та їхній порядковий номер у зображенні гіперкомплексного числа, тобто гіперкомплексні числа можна представити у вигляді вектора. Однак векторно-матрична форма тут не підходить 3 огляду на те, що компоненти матриці та вектора повинні бути однотипними. Також у системі Maple $\epsilon$ така форма представлення даних як список - list - упорядкований набір різнотипних даних. Для оперування з даними у форматі списків у Maple існують численні команди, які дозволяють задавати список, визначати довжину списку, складати два списки однакової довжини, визначати член списку за його порядковим номером у списку, множити всі члени списку на будь-який вираз тощо. Подання гіперкомплексних чисел у вигляді списку називається списочним або внутрішнім поданням гіперкомплексних чисел. Таким чином, замість (14) будемо користуватися представленням

$$
A=\left[a_{1}, a_{2}, \ldots, a_{n}\right] .
$$

Тоді сума двох чисел буде визначатися дуже просто:

$$
C=A+B=\left[a_{1}, a_{2}, \ldots, a_{n}\right]+\left[b_{1}, b_{2}, \ldots, b_{n}\right]=\left[a_{1}+b_{1}, \ldots, a_{n}+b_{n}\right],
$$

тобто приведення подібних символьних коефіцієнтів відповідно до їх порядкових номерів у числах виконується автоматичними внутрішніми засобами Maple.

Таким чином, представлення гіперкомплексних чисел у форматі списків значно спрощує розробку програмних засобів. Однак таке рішення вимагає наявності в ПКГО процедур для взаємно-зворотного перетворення натуральної і внутрішньої форм представлення гіперкомплексних чисел. Тим більше, деякі дії доцільно виконувати над числами в натуральній формі. У зв'язку з цим у багатьох процедурах ПКГО передбачається вихід у формі списку з двох елементів: перший елемент результат у списочній формі, другий - у натуральній. Так, наприклад, ось як виглядає робота процедури генерації гіперкомплексного числа восьмої вимірності:

$$
\begin{aligned}
& >A=H N \text { Snumber }(8, a, e): \\
& >A=[1] \quad\left[a_{1}, a_{2}, a_{3}, a_{4}, a_{5}, a_{6}, a_{7}, a_{8}\right] \\
& >A=[2] \quad \begin{array}{l}
> \\
a_{1} e_{1}+a_{2} e_{2}+a_{3} e_{3}+a_{4} e_{4}+a_{5} e_{5}+a_{6} e_{6}+a_{7} e_{7}+a_{8} e_{8}
\end{array}
\end{aligned}
$$

Також виявилося доцільним надати списочний формат і більш складним гіперкомплексним структурам. Так, таблиця Келі множення базисних елементів представляється трирівневою списочною структурою: верхній рівень складається зі списку рядків таблиці, другий вкладений рівень - список елементів таблиці, третій, найнижчий рівень - список структурних констант однієї клітинки таблиці Келі. 
Структурно ПКГО складається з наступних підсистем:

- виконання алгебраїчних операцій у ГЧС;

— маніпуляцій із ГЧС і таблицями Келі;

- визначення алгебраїчних характеристик гіперкомплексних виразів;

- зберігання часто вживаних виразів;

— виконання модульних операцій із гіперкомплексними виразами;

— візуалізації і сервісу.

Підсистема виконання алгебраїчних операцій у ГЧС складається з таких процедур: генерації гіперкомплексних чисел; додавання двох гіперкомплексних чисел у списочному вигляді; додавання двох гіперкомплексних чисел у натуральному вигляді; віднімання двох гіперкомплексних чисел у натуральній формі; множення двох чисел у вигляді списку; множення чисел у натуральному вигляді; ділення гіперкомплексних чисел у списочному вигляді; знаходження квадратного кореня 3 гіперкомплексних чисел у будь-якій формі; вирішення гіперкомплексного квадратного рівняння; прискореного множення комплексних чисел; прискореного множення дійсно-комплексних чисел $\mathrm{R} \oplus \mathrm{C}$.

Підсистема маніпуляцій з ГЧС і таблицями Келі складається з процедур: візуалізації списку ГЧС у таблицю Келі з даним базисом; визначення ідентифікатора базису ГЧС за гіперкомплексним числом у натуральному вигляді; перейменування ідентифікатора базису в гіперкомплексному числі в натуральному вигляді; переліку таблиць Келі ГЧС; пошуку таблиці Келі за назвою; перегляд усіх ГЧС у натуральному вигляді; транспозиції рядків і стовпців таблиці Келі ГЧС; поповнення переліку ГЧС; генерації ізоморфної ГЧС шляхом лінійного перетворення базису; побудови прямої суми двох ГЧС; побудови прямої суми декількох ГЧС; множення розмірності ГЧС; генерації системи рівнянь ізоморфізму двох ГЧС; процедури визначення ізоморфізму двох ГЧС.

Підсистема визначення алгебраїчних характеристик гіперкомплексних виразів складається з наступних процедур: визначення псевдонорми гіперкомплексного числа в списочному вигляді; визначення одиничного елемента ГЧС; побудови спряженого числа; обчислення детермінанта від гіперкомплексних матриць.

Така структура і склад ПКГО в середовищі Maple, як буде показано далі, дозволяє значно спростити процеси створення програмного забезпечення для математичного моделювання різних науково-технічних задач.

Усі процедури ПКГО можна використовувати при написанні програм вирішення різних задач з гіперкомплексними даними мовою програмування Maple. Для цього треба підключити до програми ПКГО відповідно до вимог CКА Maple та викликати потрібні процедури за їхніми ідентифікаторами, як це буде показано далі.

\section{Характеристики класу досліАжуваних ГЧС}

Як зазначає O.I. Кострікін у [12], «Задача вивчення довільних алгебраїчних структур дуже загальна, щоби вона являла реальну цінність. Тому їі розглядають при різноманітних природніх обмеженнях». Оскільки клас різноманітних ГЧС дуже широкий, обмежимося в даній роботі розглядом таких ГЧС, які являють собою групові алгебри [12], що їхні базисні елементи створюють групи $G$, ізоморфні адитивним групам $Z_{n}$ класів лишків за цілочисельним модулем $n$, тобто 


$$
G \simeq Z_{n}=Z / n Z .
$$

Групова операція, або закон композиції базисних елементів, виражається формулою:

$$
e_{i} \cdot e_{j}=e_{(i+j)(\bmod n)}, i, j=0,1, \ldots, n-1,
$$

де $n$ фактично є вимірність досліджуваної ГЧС.

Як випливає з (16), такі ГЧС є комутативними і асоціативними. Саме це визначає практичну цінність їхнього використання для моделювання.

Оскільки в наших роботах більш зручно визначати індекси базисних елементів від 1 до $n$, то (16) трансформується у формулу:

$$
e_{i} \cdot e_{j}=e_{(i+j-2)(\bmod n)+1}, i, j=1, \ldots, n .
$$

Слід зазначити, що при $n \leq 4$ такі ГЧС вже досліджені.

Так, при $n=2$ - це система подвійних чисел, яка в класифікації ПКГО має позначення $W$, і таблиця Келі якої має вигляд:

\begin{tabular}{|l|l|l|}
\hline$W$ & $e_{1}$ & $e_{2}$ \\
\hline$e_{1}$ & $e_{1}$ & $e_{2}$ \\
\hline$e_{2}$ & $e_{2}$ & $e_{1}$ \\
\hline
\end{tabular}

ГЧС $W$ ізоморфна системі подвійно-ортогональних чисел $W 1=R \oplus R$. Представлення експоненти в системі $W$ виглядає так:

$$
\operatorname{Exp}\left(m_{1} e_{1}+m_{2} e_{2}\right)=e^{m_{1}}\left(\cosh m_{2} \cdot e_{1}+\sinh m_{2} \cdot e_{2}\right) .
$$

Ця ГЧС комутативна та асоціативна.

При $n=3$ одержуємо ГЧС $G_{33}$ [8], таблиця Келі якої має вигляд:

\begin{tabular}{|l|l|l|l|}
\hline$G_{33}$ & $e_{1}$ & $e_{2}$ & $e_{3}$ \\
\hline$e_{1}$ & $e_{1}$ & $e_{2}$ & $e_{3}$ \\
\hline$e_{2}$ & $e_{2}$ & $e_{3}$ & $e_{1}$ \\
\hline$e_{3}$ & $e_{3}$ & $e_{1}$ & $e_{2}$ \\
\hline
\end{tabular}

Ця ГЧС ізоморфна системам дійсно-комплексних чисел $R \oplus C$ та триплексних чисел $T$ :

$$
G_{33} \simeq R \oplus C \simeq T .
$$

Крім того, слід зазначити, що мультиплікативна підгрупа ГЧС $G_{33}$ ізоморфна знакозмінній підгрупі $A_{3}$ симетричної групи $S_{3}$. ГЧС $G_{33}$ комутативна та асоціативна.

Представлення експоненти в системі $G_{33}$ виглядає так: 


$$
\begin{aligned}
\operatorname{Exp}\left(m_{1} e_{1}+m_{2} e_{2}+m_{3} e_{3}\right)= & \left(e^{\alpha}+2 e^{\beta} \cos \gamma\right) e_{1}+\left(e^{\alpha}-e^{\beta} \cos \gamma+\sqrt{3} e^{\beta} \sin \gamma\right) e_{2}+ \\
& +\left(e^{\alpha}-e^{\beta} \cos \gamma-\sqrt{3} e^{\beta} \sin \gamma\right) e_{3},
\end{aligned}
$$

де $\alpha=m_{1}+m_{2}+m_{3}, \quad \beta=m_{1}-m_{2} / 2-m_{3} / 2, \gamma=\sqrt{3}\left(m_{2}-m_{3}\right)$.

Якщо представлення експоненти (18) в ГЧС $W$ можна легко отримати за алгоритмом за формулами (4)-(10), то побудова представлення в $Г Ч С ~ G 3$ значно складніше. Воно потребує істотного використання засобів ПКГО.

Розглянемо випадок $n=4$ : використовуємо ГЧС $G_{47}$ за класифікацією ПКГО, таблиця Келі якої має вигляд:

\begin{tabular}{|l|l|l|l|l|}
\hline$G_{47}$ & $e_{1}$ & $e_{2}$ & $e_{3}$ & $e_{4}$ \\
\hline$e_{1}$ & $e_{1}$ & $e_{2}$ & $e_{3}$ & $e_{4}$ \\
\hline$e_{2}$ & $e_{2}$ & $e_{3}$ & $e_{4}$ & $e_{1}$ \\
\hline$e_{3}$ & $e_{3}$ & $e_{4}$ & $e_{1}$ & $e_{2}$ \\
\hline$e_{4}$ & $e_{4}$ & $e_{1}$ & $e_{2}$ & $e_{3}$ \\
\hline
\end{tabular}

Можна показати, що мультиплікативна підгрупа ГЧС $G_{47}$ ізоморфна підгрупі $V=\{e,(1234),(13)(24),(1432)\}$, яка являє нормальну підгрупу симетричної групи $S_{4}: V \triangleleft S_{4}$.

Характеристичне рівняння (9) має два дійсних і пару комплексно-спряжених коренів:

$$
\lambda_{1,2}=m_{1}+m_{3} \pm\left(m_{2}+m_{4}\right), \lambda_{3,4}=m_{1}-m_{3} \pm i\left(m_{2}-m_{4}\right) .
$$

3 цього факту можна зробити висновок, що ГЧС має принаймні дві ізоморфних системи [13]: $R \oplus R \oplus C$ та $W \oplus C$, таблиці Келі яких приводяться нижче:

\begin{tabular}{|c|l|l|l|l|}
\hline$R \oplus R \oplus C$ & $e_{1}$ & $e_{2}$ & $e_{3}$ & $e_{4}$ \\
\hline$e_{1}$ & $e_{1}$ & 0 & 0 & 0 \\
\hline$e_{2}$ & 0 & $e_{3}$ & 0 & 0 \\
\hline$e_{3}$ & 0 & 0 & $e_{3}$ & $e_{4}$ \\
\hline$e_{4}$ & 0 & 0 & $e_{4}$ & $-e_{3}$ \\
\hline
\end{tabular}

\begin{tabular}{|c|l|l|l|l|}
\hline$W \oplus C$ & $e_{1}$ & $e_{2}$ & $e_{3}$ & $e_{4}$ \\
\hline$e_{1}$ & $e_{1}$ & $e_{2}$ & 0 & 0 \\
\hline$e_{2}$ & $e_{2}$ & $e_{1}$ & 0 & 0 \\
\hline$e_{3}$ & 0 & 0 & $e_{3}$ & $e_{4}$ \\
\hline$e_{4}$ & 0 & 0 & $e_{4}$ & $-e_{3}$ \\
\hline
\end{tabular}

Якщо ввести позначення

$$
\alpha_{1,2}=m_{1} \pm m_{3}, \quad \beta_{1,2}=m_{3} \pm m_{4},
$$

то представлення експоненти в системі $G_{47}$ виглядає так: 


$$
\begin{gathered}
\operatorname{Exp}\left(m_{1} e_{1}+m_{2} e_{2}+m_{3} e_{3}+m_{4} e_{4}\right)= \\
=\frac{1}{2}\left[\left(e^{\alpha_{1}} \cosh \beta_{1}+e^{\alpha_{2}} \cos \beta_{2}\right) e_{1}+\left(e^{\alpha_{1}} \sinh \beta_{1}+e^{\alpha_{2}} \sin \beta_{2}\right) e_{2}+\right. \\
\left.+\left(e^{\alpha_{1}} \cosh \beta_{1}-e^{\alpha_{2}} \cos \beta_{2}\right) e_{3}+\left(e^{\alpha_{1}} \sinh \beta_{1}-e^{\alpha_{2}} \sin \beta_{2}\right) e_{4}\right] .
\end{gathered}
$$

ГЧС $G_{47}$ комутативна та асоціативна.

\section{Побудова експоненти в ГЧС вимірності $n=5$}

Як показано вище, дії з гіперкомплексними виразами, особливо в ГЧС високих вимірностей та ще й у символьній формі, дуже громіздкі. Тому всі розрахунки та перетворення в ГЧС п'ятої вимірності $G_{51}$ будемо виконувати з використанням ПКГО. Це дозволить як зменшити витрати часу, так і уникнути помилок. Наші міркування будемо супроводжувати фрагментами програм у середовищі мови СКА Maple з використанням засобів ПКГО.

Викликаємо зі сховища гіперкомплексних числових систем LibHNS() ГЧС і відображаємо іiі таблицю Келі:

$$
\begin{aligned}
& \text { G51 := SearchHNS }(\text { G51, LibHNS( ) ) : } \\
& \operatorname{VizHNS}(\text { G51,e) } \\
& {\left[\begin{array}{lllll}
e_{1} & e_{2} & e_{3} & e_{4} & e_{5} \\
e_{2} & e_{3} & e_{4} & e_{5} & e_{1} \\
e_{3} & e_{4} & e_{5} & e_{1} & e_{2} \\
e_{4} & e_{5} & e_{1} & e_{2} & e_{3} \\
e_{5} & e_{1} & e_{2} & e_{3} & e_{4}
\end{array}\right]}
\end{aligned}
$$

Для побудови матриці правої частин (4) треба виконати гіперкомплексне множення і перетворити гіперкомплексне рівняння (4) в систему дійсних рівнянь, після чого можна безпосередньо побудувати матрицю і знайти ії характеристичні числа. Засобами ПКГО це виглядає так:

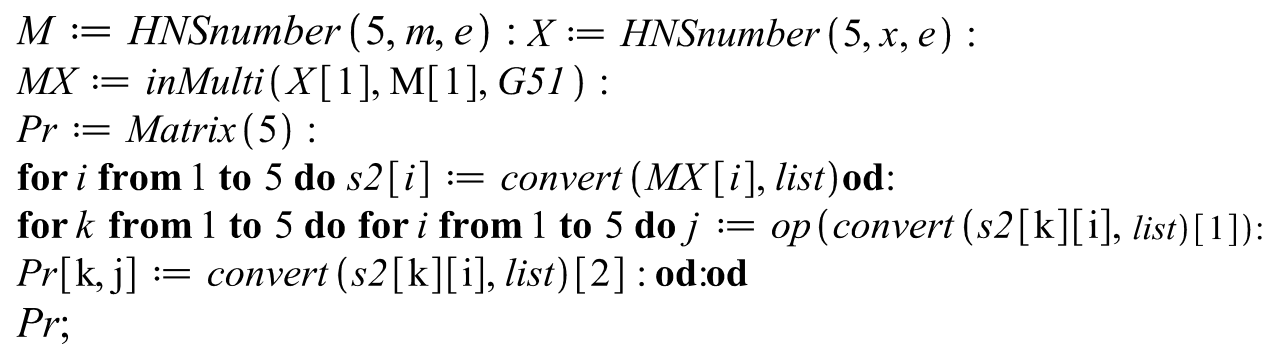




$$
\left[\begin{array}{ccccc}
m_{1} & m_{5} & m_{4} & m_{3} & m_{2} \\
m_{2} & m_{1} & m_{5} & m_{4} & m_{3} \\
m_{3} & m_{2} & m_{1} & m_{5} & m_{4} \\
m_{4} & m_{3} & m_{2} & m_{1} & m_{5} \\
m_{5} & m_{4} & m_{3} & m_{2} & m_{1}
\end{array}\right]
$$

Шукаємо характеристичні числа матриці $\mathrm{Pr}$ :

$$
\lambda:=\operatorname{Eigenvalues}(\operatorname{Pr}) \text { : }
$$

Назвемо набір характеристичних чисел для конкретної ГЧС ії спектром. Перше з них явно дійсне:

$$
\lambda_{1}=m_{2}+m_{3}+m_{4}+m_{5}+m_{1},
$$

а інші чотири корені утворюють дві пари такого вигляду:

$$
a \pm \sqrt{b} \text {. }
$$

Для другого та третього коренів

$$
\begin{gathered}
a=m_{1}-\frac{1}{4} m_{5}-\frac{1}{4} m_{2}-\frac{1}{4} m_{3}-\frac{1}{4} m_{4}, \\
b=\left(-2 \sqrt{5} m_{2}^{2}-10 m_{2}^{2}+4 \sqrt{5} m_{5} m_{2}+20 m_{5} m_{2}-8 \sqrt{5} m_{3} m_{2}+\right. \\
+8 \sqrt{5} m_{4} m_{2}-10 m_{5}^{2}+2 \sqrt{5} m_{4}^{2}+ \\
+2 \sqrt{5} m_{3}^{2}-8 \sqrt{5} m_{4} m_{5}-10 m_{4}^{2}-2 \sqrt{5} m_{5}^{2}- \\
\left.-m_{3}^{2}+20 m_{3} m_{4}+8 \sqrt{5} m_{3} m_{5}-4 \sqrt{5} m_{3} m_{4}\right) / 4 .
\end{gathered}
$$

Якщо $a$ - число дійсне, то від знаку $b$ залежить, чи будуть корені (20) дійсними, чи комплексними. I взагалі, невідомо, чи буде $b$ мати постійний знак. Якщо $b \geq 0$, то корінь - дійсне число, як сума і різниця двох дійсних чисел. Якщо ж $b<0$, то корінь комплексний.

Однак, за зовнішнім виглядом дуже важко судити про знак виразу $b$. Крім того, в принципі, він може мати різні знаки в залежності від значень коефіцієнтів. Зауважимо, однак, що якщо буде так, то це буде свідчити про помилку в обчисленнях, тому що, як показано в [13], тип спектра не змінюється при лінійному перетворенні базису ГЧ.

Вираз $b$ - це квадратична форма від 4-х змінних $-m_{2}, m_{3}, m_{4}, m_{5}$, матриця якої у даному випадку має вигляд:

$$
K 1:=\left|\begin{array}{rrrr}
-2 \sqrt{5}-10 & -4 \sqrt{5} & 4 \sqrt{5} & 2 \sqrt{5}+10 \\
-4 \sqrt{5} & -10+2 \sqrt{5} & +10-2 \sqrt{5} & 4 \sqrt{5} \\
4 \sqrt{5} & +10-2 \sqrt{5} & 2 \sqrt{5}-10 & -4 \sqrt{5} \\
2 \sqrt{5}+10 & 4 \sqrt{5} & -4 \sqrt{5} & -10-2 \sqrt{5}
\end{array}\right|
$$


Квадратичні форми можуть бути знаковизначеними, тобто їхні значення приймають певний знак («+» або «-») при будь-яких значеннях аргументів квадратичної форми, або знакозмінними в протилежному випадку.

Для визначення типу квадратичної форми існують різні критерії [14]. Основним критерієм $є$ критерій Сильвестра, відповідно до якого вивчаються знаки головного мінору матриці квадратичної форми. В даному випадку всі головні мінори дорівнюють нулю.

Тому потрібно користуватися критерієм знаків власних чисел матриці квадратичної форми. Визначаємо за допомогою процедури Eigenvalues пакета LinearAlge bra власні числа матриці квадратичної форми $K 1$. Вони будуть такими: $[0,0,0,-40]$.

Усі чотири власних числа не додатні, тобто квадратична форма не додатня. Вона може дорівнювати нулю, але нас цей випадок не цікавить. Таким чином, ці два характеристичних числа матриці - комплексно-спряжена пара.

Як бачимо цей аналіз достатньо складний. А засобами ПКГО він дуже простий, бо там $\epsilon$ процедура ARoot, яка визначає знак виразу $b$ шляхом його факторизації, що дає:

$$
b=-\frac{(10+2 \sqrt{5}}{64}\left(-2 m_{5}+2 m_{2}+\sqrt{5} m_{3}-\sqrt{5} m_{4}-m_{3}+m_{4}\right)^{2}<0,
$$

звідки випливає, що характеристичні числа $\lambda_{2}, \lambda_{3}$ матриці $\operatorname{Pr}$ - це комплексноспряжена пара.

Аналогічно можна визначити, що і характеристичні числа $\lambda_{4}, \lambda_{5}$ теж являють собою комплексно-спряжену пару. Приведемо вигляд останніх чотирьох характеристичних чисел:

$$
\begin{gathered}
\lambda_{2,3,4,5}=\left(\frac{1}{4}\left(m_{2}+m_{3}+m_{4}+m_{5}\right) \pm \frac{\sqrt{5}}{4}\left(m_{2}-m_{3}-m_{4}+m_{5}\right)\right) \pm \\
\pm I\left( \pm 2 m_{2} \mp 2 m_{5} \mp m_{3} \pm m_{4}+\sqrt{5}\left(m_{3}-m_{4}\right)\right) \frac{\sqrt{10 \pm \sqrt{5}}}{8} .
\end{gathered}
$$

Таким чином, матриця правої частини асоційованої системи лінійних диференціальних рівнянь для ГЧС $G_{51}$ має одне дійсне характеристичне число і дві різні пари комплексно-спряжених. Тому можна визначити, що ГЧС $G_{51}$ ізоморфна прямій сумі двох систем дійсних чисел $R$ та системи комплексних чисел $C$ :

$$
G_{51} \cong R \oplus R \oplus C \text {. }
$$

Мультиплікативна група ГЧС $G 51$, яку позначимо $G_{51}{ }^{*}$, ізоморфна такій підгрупі $V$ симетричної групи $S_{5}$ :

$$
V=\{e,(12345),(13524),(14253),(15432)\} \subset S_{5} .
$$

Оскільки підгрупа $V$ складається тільки з непарних циклів, тобто з парних підстановок, то підгрупа $є$ підгрупою знакозмінної підгрупи $A_{5} \subset S_{5}$. 
Для подальшої побудови експоненти треба побудувати часткові розв'язки системи рівнянь (8) для всіх характеристичних чисел.

Часткові розв'язки для $\lambda_{1}$ повинні мати вигляд:

$$
X p_{1 i}=C_{1 i} e^{\lambda_{1} t}, i=1, \ldots, 5 .
$$

Після підстановки в (8) і спрощення буде одержана лінійна система, з якої можна одержати значення довільних констант: $C_{1 i}=i d e m, i=1, \ldots, 5$, тобто, залишиться тільки одна довільна константа.

Для першої пари комплексно-спряжених характеристичних чисел $\lambda_{2,3}$ часткові розв'язки повинні мати вигляд:

$$
X p_{2 i}=e^{r e \lambda_{2} t}\left(C_{2 i} \cos \left(i m \lambda_{2} \cdot t\right)+F_{2 i} \sin \left(i m \lambda_{2} \cdot t\right)\right), \quad i=1, \ldots, 5 .
$$

Підстановка цих розв'язків у рівняння (4) за допомогою процедури ПКГО Const 1 дає систему з 5-ти дійсних лінійних рівнянь. Оскільки ці рівняння дуже громіздкі, приведемо для ілюстрації лише одне з них:

$$
\begin{aligned}
& -\frac{1}{4} m_{2} F_{2,1}-m_{5} F_{2,2}-\frac{1}{4} m_{4} F_{2,1}-m_{4} F_{2,3}-\frac{1}{4} m_{3} F_{2,1}-m_{3} F_{2,4}-\frac{1}{4} m_{5} F_{2,1}-m_{2} F_{2,5}+ \\
& +\frac{1}{8} C_{2,1} \sqrt{10+2 \sqrt{5}} * \sqrt{5} m_{4}-\frac{1}{8} C_{2,1} \sqrt{10+2 \sqrt{5}} * \sqrt{5} m_{3}+\frac{1}{4} \sqrt{5} m_{2} F_{2,1}-\frac{1}{4} \sqrt{5} m_{3} F_{2,1}- \\
& -\frac{1}{-4} \sqrt{5} m_{4} F_{2,1}+\frac{1}{4} \sqrt{5} m_{5} F_{2,1}-\frac{1}{8} C_{2,1} \sqrt{10+2 \sqrt{5}} * m_{4}+\frac{1}{8} C_{2,1} \sqrt{10+2 \sqrt{5}} * m_{3}+ \\
& +\frac{1}{4} C_{2,1} \sqrt{10+2 \sqrt{5}} * m_{5}-\frac{1}{4} C_{2,1} \sqrt{10+2 \sqrt{5}} * m_{2}=0 .
\end{aligned}
$$

Результати вирішення цієї системи за допомогою Maple-процедури solve дуже громіздкі. Наприклад, для довільної константи воно має вигляд:

$$
\begin{aligned}
& C_{2,1}=-\left(-C_{2,3} m_{5}^{2}+C_{2,3} m_{3}^{2}-C_{2,3} m_{2}^{2}+C_{2,3} m_{4}^{2}+\sqrt{5} C_{2,4} m_{3}^{2}+\sqrt{5} C_{2,4} m_{4}^{2}+C_{2,4} m_{2} m_{3}-\right. \\
& -C_{2,4} m_{2} m_{4}+3 C_{2,4} m_{4} m_{3}-C_{2,4} m_{5} m_{3}-3 C_{2,4} m_{5} m_{2}+C_{2,4} m_{5} m_{4}+\sqrt{5} C_{2,4} m_{2}^{2}+ \\
& +\sqrt{5} C_{2,4} m_{5}^{2}+\sqrt{5} C_{2,3} m_{5} m_{4}+\sqrt{5} C_{2,3} m_{5} m_{2}+\sqrt{5} C_{2,3} m_{5} m_{3}+\sqrt{5} C_{2,3} m_{3} m_{4}+ \\
& +\sqrt{5} C_{2,3} m_{2} m_{4}+\sqrt{5} C_{2,3} m_{2} m_{3}-C_{2,4} m_{5}^{2}-C_{2,4} m_{2}^{2}+C_{2,4} m_{3}^{2}+C_{2,4} m_{4}^{2}+\sqrt{5} C_{2,3} m_{3}^{2}+ \\
& +\sqrt{5} C_{2,3} m_{4}^{2}+\sqrt{5} C_{2,3} m_{2}^{2}-3 C_{2,3} m_{2} m_{5}+C_{2,3} m_{2} m_{3}-C_{2,3} m_{3} m_{5}+C_{2,3} m_{4} m_{5}-C_{2,3} m_{2} m_{4}+ \\
& +3 C_{2,3} m_{3} m_{4}+\sqrt{5} C_{2,3} m_{5}^{2}+\sqrt{5} C_{2,4} m_{2} m_{5}+\sqrt{5} C_{2,4} m_{3} m_{4}+\sqrt{5} C_{2,4} m_{3} m_{2}+\sqrt{5} C_{2,4} m_{5} m_{4}+ \\
& \left.+\sqrt{5} C_{2,4} m_{2} m_{4}+\sqrt{5} C_{2,4} m_{3} m_{5}\right) /\left(2 m_{5}^{2}+2 m_{5} m_{2}-\sqrt{5} m_{5} m_{2}+3 m_{5} m_{4}+\sqrt{5} m_{5} m_{4}+\right. \\
& +m_{5} m_{2}+3 m_{3}^{2}+3 m_{4}^{2}+\sqrt{5} m_{4}^{2}+\sqrt{5} m_{3}^{2}+2 m_{4} m_{2}+\sqrt{5} m_{3} m_{2}+2 m_{2}^{2}+4 m_{4} m_{3}+ \\
& \left.+2 \sqrt{5} m_{3} m_{4}+3 m_{3} m_{2}\right) .
\end{aligned}
$$

Цей вираз потребує значного спрощення, яке в автоматичному режимі не діє. Вираз являє собою дріб. Тому спрощення можливе, коли чисельник і знаменник факторизувати окремо, і тоді вони автоматично скорочуються на загальний множник. Ці операції можна виконати за допомогою такої конструкції:

$$
C[2,1]:=\frac{\text { factor }(\text { numer }(C[2,1]))}{\text { factor }(\text { denom }([2,1]))},
$$

що дає дуже простий вираз:

$$
C_{2,1}:=\frac{1}{2} C_{2,5} \sqrt{5}-\frac{1}{2} C_{2,5}-C_{2,4}
$$


Як випливає з теорії лінійних диференціальних рівнянь, в одержаній системі 5-ти рівнянь 3 10-ма змінними тільки дві змінні лінійно незалежні. I дійсно, результат вирішення цієї системи за допомогою Мaple-процедури solve дає такі результати:

$$
\begin{aligned}
& C_{2,1}:=\frac{1}{2} C_{2,5} \sqrt{5}-\frac{1}{2} C_{2,5}-C_{2,4}, \\
& C_{2,2}:=-\frac{1}{2}(\sqrt{5}-1)\left(C_{2,5}+C_{2,4}\right), \\
& C_{2,3}:=-\frac{1}{4}(\sqrt{5}-1)\left(C_{2,5}+\sqrt{5} C_{2,5}-2 C_{2,4}\right), \\
& C_{2,4}:=C_{2,4}, \\
& C_{2,5}:=C_{2,5}, \\
& F_{2,1}:=\frac{1}{2} * \frac{(\sqrt{5}-1)\left(C_{2,5} \sqrt{5}+3 C_{2,5}+2 C_{2,4}\right)}{\sqrt{10+2 \sqrt{5}}}, \\
& F_{2,2}:=-\frac{(\sqrt{5}+1)\left(-C_{2,5}+C_{2,4}\right)}{\sqrt{10+2 \sqrt{5}}}, \\
& F_{2,3}:=\frac{1}{2} * \frac{(\sqrt{5}+1)\left(C_{2,5} \sqrt{5}-3 C_{2,5}-2 C_{2,4}\right)}{\sqrt{10+2 \sqrt{5}}}, \\
& F_{2,4}:=-\frac{(\sqrt{5}-1)\left(C_{2,5} \sqrt{5}+C_{2,5}-C_{2,4}\right)}{\sqrt{10+2 \sqrt{5}}}, \\
& F_{2,5}:=-\frac{\left.C_{2,5} \sqrt{5}-C_{2,5}-4 C_{2,4}\right)}{\sqrt{10+2 \sqrt{5}}}
\end{aligned}
$$

Як видно зі співвідношень (23), лінійно незалежними константами є константи $C_{24}$ та $C_{25}$, а решта констант лінійно залежать від них.

Повністю аналогічно можна одержати і залежності для констант другої пари комплексно-спряжених характеристичних чисел:

$$
\begin{aligned}
& C_{4,1}:=-\frac{1}{2} C_{4,5} \sqrt{5}-\frac{1}{2} C_{4,5}-C_{4,4}, \\
& C_{4,2}:=\frac{1}{2}(\sqrt{5}+1)\left(C_{4,5}+C_{4,4}\right), \\
& C_{4,3}:=-\frac{1}{4}(\sqrt{5}+1)\left(-C_{4,5}+\sqrt{5} C_{4,5}+2 C_{4,4}\right), \\
& C_{4,4}:=C_{4,4}, \\
& C_{4,5}:=C_{4,5}, \\
& F_{4,1}:=-\frac{1}{4} * \frac{(\sqrt{5}+1)\left(C_{4,5} \sqrt{5}-3 C_{4,5}-2 C_{4,4}\right)}{\sqrt{5-\sqrt{5}}}, \\
& F_{4,2}:=-\frac{1}{2} * \frac{\sqrt{2}(\sqrt{5}-1)\left(-C_{4,5}+C_{4,4}\right)}{\sqrt{5-\sqrt{5}}}, \\
& F_{4,3}:=-\frac{1}{4} * \frac{\sqrt{2}(\sqrt{5}-1)\left(C_{4,5} \sqrt{5}-3 C_{4,5}-2 C_{4,4}\right)}{\sqrt{5-\sqrt{5}}}, \\
& F_{4,4}:=\frac{1}{2} * \frac{\sqrt{2}(\sqrt{5}+1)\left(C_{4,5} \sqrt{5}-C_{4,5}+C_{4,4}\right)}{\sqrt{5-\sqrt{5}}} \\
& F_{4,5}:=-\frac{1}{2} * \frac{\sqrt{2}\left(C_{4,5} \sqrt{5}+C_{4,5}+4 C_{4,4}\right)}{\sqrt{5-\sqrt{5}}}
\end{aligned}
$$

Слід відзначити, що вибір незалежних констант здійснює сама програма, і в різних сеансах їхній склад може змінюватися, що може надати деяких незручностей. Але можна так побудувати алгоритм вирішення, що ці незручності еліміну- 
ються. Саме так і побудовано алгоритм, який використовується в даній роботі. Звичайно, можна було призначати ці константи попередньо, але, як показує досвід роботи з Maple-командою solve, автоматичний вибір дає розв'язки найпростішого вигляду.

Далі потрібно побудувати 5 компонент загального розв'язку системи рівнянь (4), які представляють собою суми часткових розв'язків - по одному вигляду (21) і по два вигляду (22), які будуть залежати від п'яти незалежних констант.

Для визначення цих констант треба врахувати початкову умову для (4): в початковий момент всі компоненти розв'язку, крім першого, приймуть нульове значення, перший компонент - одиничне значення, тобто як для експоненти від дійсного аргументу. При цьому одержимо таку систему з п’яти рівнянь:

$$
\begin{gathered}
C_{1,5}-\frac{1}{2}(\sqrt{5}-1)\left(C_{2,4}+C_{2,3}\right)+\frac{1}{2}(1+\sqrt{5})\left(C_{4,4}+C_{4,3}\right)=1, \\
C_{1,5}-\frac{1}{4}(\sqrt{5}-1)\left(C_{2,4}+C_{2,4} \sqrt{5}-2 C_{2,3}\right)-\frac{1}{4}(1+\sqrt{5})\left(C_{4,4} \sqrt{5}-C_{4,4}+2 C_{4,3}\right)=0, \\
C_{1,5}+C_{2,3}+C_{4,3}=0, \\
C_{1,5}+C_{2,4}+C_{4,4}=0, \\
C_{1,5}+\frac{1}{2} C_{2,4} \sqrt{5}-\frac{1}{2} C_{2,4}-C_{2,3}-\frac{1}{2} C_{4,4}-\frac{1}{2} C_{4,4} \sqrt{5}-C_{4,3} 1=0 .
\end{gathered}
$$

Після вирішення цієї системи, підстановки значень незалежних констант до (22) i (23) одержимо значення всіх 25-ти констант, які приведено в таблиці.

Константи інтегрування

\begin{tabular}{||l||c||c||c||c||c||}
\hline \hline & $C_{1 i}$ & $C_{2 i}$ & $F_{2 i}$ & $C_{3 i}$ & $F_{3 i}$ \\
\hline \hline 1 & $1 / 5$ & $2 / 5$ & 0 & $2 / 5$ & 0 \\
\hline 2 & $1 / 5$ & $\frac{1}{10} \sqrt{5}-\frac{1}{10}$ & $\frac{1}{5} \frac{5+\sqrt{5}}{\sqrt{10+2 \sqrt{5}}}$ & $-\frac{1}{10} \sqrt{5}-\frac{1}{10}$ & $-\frac{1}{10} \sqrt{2} \sqrt{5-\sqrt{5}}$ \\
\hline 3 & $1 / 5$ & $-\frac{1}{10} \sqrt{5}-\frac{1}{10}$ & $\frac{2}{5} \frac{\sqrt{5}}{\sqrt{10+2 \sqrt{5}}}$ & $\frac{1}{10} \sqrt{5}-\frac{1}{10}$ & $\frac{1}{5} \frac{\sqrt{2} \sqrt{5}}{\sqrt{5-\sqrt{5}}}$ \\
\hline 4 & $1 / 5$ & $-\frac{1}{10} \sqrt{5}-\frac{1}{10}$ & $\frac{2}{5} \frac{\sqrt{5}}{\sqrt{10+2 \sqrt{5}}}$ & $\frac{1}{10} \sqrt{5}-\frac{1}{10}$ & $\frac{1}{5} \frac{\sqrt{2} \sqrt{5}}{\sqrt{5-\sqrt{5}}}$ \\
\hline 5 & $1 / 5$ & $\frac{1}{10} \sqrt{5}-\frac{1}{10}$ & $-\frac{1}{5} \frac{5+\sqrt{5}}{\sqrt{10+2 \sqrt{5}}}$ & $-\frac{1}{10} \sqrt{5}-\frac{1}{10}$ & $\frac{1}{10} \sqrt{2} \sqrt{5-\sqrt{5}}$ \\
\hline
\end{tabular}

Тепер можна записати кінцеву формулу представлення експоненти в ГЧС $G_{51}:$

$$
\begin{aligned}
\operatorname{Exp}(M) & =\sum_{i=1}^{5}\left(\frac{1}{5} e^{\lambda_{1}}+e^{r e \lambda_{2}}\left(C_{2 i} \cos \left(i m \lambda_{2}\right)+F_{2 i} \sin \left(i m \lambda_{2}\right)+\right.\right. \\
& +e^{r e \lambda_{4}}\left(C_{3 i} \cos \left(i m \lambda_{4}\right)+F_{3 i} \sin \left(i m \lambda_{4}\right) e_{i}\right),
\end{aligned}
$$


де

$$
\begin{gathered}
M=\sum_{i=1}^{5} m_{i} e_{i}, \\
\lambda_{1}=\sum_{i-1}^{5} m_{i}, \\
r e \lambda_{2,4}=\frac{\sqrt{10 \pm \sqrt{5}}}{32}\left(\left(m_{2}+m_{3}+m_{4}+m_{5}\right) \pm \sqrt{5}\left(m_{2}-m_{3}-m_{4}+m_{5}\right)\right), \\
i m \lambda_{2,4}=\frac{\sqrt{10 \pm \sqrt{5}}}{8}\left(\left( \pm 2 m_{2} \mp 2 m_{5} \mp m_{3} \pm m_{4}+\sqrt{5}\left(m_{3}-m_{4}\right)\right) .\right.
\end{gathered}
$$

Висновки

1. Метод асоційованої з ГЧС системи лінійних диференціальних рівнянь дозволяє будувати представлення експонент в системах високих вимірностей.

2. Програмний комплекс гіперкомплексних обчислень показав високу ефективність при оперуванні з громіздкими символьними виразами і розв'язуванні лінійних і нелінійних рівнянь з гіперкомплексними коефіцієнтами.

1. Hamilton W. R. Researches respecting quaternions: first series. Transactions of the Royal Irish Academy. 1848. 21, Part 1. P. 199-296.

2. Brackx F. The exponential function of a quaternion variable. Applicable Analysis. 1979. 8. P. 265- 276.

3. Scheicher K., Tichy R.F., Tomantschger K.W. Elementary Inequalities in Hypercomplex Numbers Anzeiger. 1997. Abt. II, No. 134. P. 3-10.

4. Калиновский Я.А., Роенко Н.В., Синьков М.В. Методы построения нелинейностей в расширениях комплексных чисел. Кибернетика и системный анализ. 1996. № 4. С. 178-181.

5. Калиновский Я.А., Синьков М.В., Роенко Н.В. Building nonlinear functions in quaternion and other hypercomplex number systems for the solution of applied mecanics problem. Proc. of the First Int. Conf. On parallel processing and appl. Math. Poland, 1994. P. 170-177.

6. Синьков М.В., Калиновский Я.А, Бояринова Ю.Е., Федоренко А.Ф. О дифференциальных уравнениях, определяющих функции гиперкомплексного переменного. Реєстрація, зберігання і оброб. даних. 2006. Т. 8, № 3. С. 20-24.

7. Калиновський Я.А., Синьков М.В., Бояринова Ю.С., Федоренко О.В. Зображення нелінійностей в скінченновимірних гіперкомплексних числових системах. Доповіді НАНУ. 2008. № 8. C. $43-51$.

8. Калиновский Я.А., Синьков М.В., Бояринова Ю.Е. Конечномерные гиперкомплексные числовые системы. Основы теории. Применения. Киев: Инфодрук, 2010. 388 с.

9. Клименко В.П., Ляхов А.Л., Гвоздик Д.Н. Современные особенности развития систем компьютерной алгебры. Математичні машини і системи. 2011. № 2. С. 3-18.

10. Татарников О. Обзор программ для символьной математики. URL: https://compress.ru /article.aspx?id=16152

11. Калиновский Я.А., Бояринова Ю.Е. Хицко Я.В. Гиперкомплексные вычисления в Maple. Киев: ИПРИ НАНУ, 2020. 180 с. ISBN 978-966-02-8879-9.

12. Кострикин А.И. Введение в алгебру. Москва: Наука, 1977. 496 с.

13. Калиновский Я.А., Бояринова Ю.Е. Высокоразмерные изоморфные гиперкомплексные числовые системы и их применения. Киев: ИПРИ НАНУ, 2012. 183 с.

14. Корн Г., Корн Т. Справочник по математике для научных работников и инженеров. Москва: Наука, 1974. 832 с.

Надійшла до редакції 20.05.2021 